\title{
THE USE OF A TREE-LIKE POROUS STRUCTURE MODEL FOR ESTIMATING OF THE IGNITION TEMPERATURE OF A CARBON RESIDUE PARTICLE
}

(C) V.A. Kvitsinsky, S.I. Kryvosheev, PhD in technical sciences, S.V. Marushchak (Institute of Coal Energy Technologies of the NAS of Ukraine, 19, Andriyvska str., 04070, Kiyiv, Ukraine)

A model of a tree-like porous structure has been used to calculate the thermal effects of chemical reactions of the molecular oxygen and carbon dioxide with an accessible particle surface of a carbon residue and to estimate heat balance with a gas environment. The effective reaction rate constant of the oxygen and carbon dioxide for the contour surface of the particle has been presented as the averaged value of the effective constants for the components of the surface of the carbon residue partricle: the inlets in the micro-, meso-, macropores; the density of outer surface; the areas of mineral impurities. The model takes into account: the volume fractions of micro-, meso-, macropores and mineral impurities in the carbon residue; the ratio of the length and radius of the pores; Knudsen diffusion in the pores; the density of branches on the surface of the transport pores; the reaction rate constant on dense carbon surface; limiting the reaction rate in the pores by the amount of chaotic flow of reagent molecules into the inlet. The external mass transfer of the carbon residue particle has been taken into account in the model of the surface layer. This factor depends on the particle diameter and the steady velocity in the gaseous environment.

When calculating the heat balance of the particles, the thermal effect of chemical reactions of the oxygen and carbon dioxide with carbon, convective heat exchange with the gaseous environment and heat loss due to radiation have been taken into account. In the stationary model, the ignition temperature of carbon residue particles in a gaseous environment containing the oxygen and carbon dioxide as components have been calculated. It has been shown that the parameters of the porous structure significantly effect on the ignition temperature and the equilibrium temperature of the particle in the flue gases. The dependence of the ignition temperature of the particles on the value of the available surface of the carbon residue has been obtained.

Keywords: carbon residue, particle, mineral impurity concentration, model of tree-like porous structure, effective reaction rate for $\mathrm{O}_{2}$ and $\mathrm{CO}_{2}$, ignition temperature. 